\title{
BOUNDEDNESS FOR THE GENERALIZED COMMUTATOR OF SJÖLIN TYPE OPERATORS
}

\section{XiAO Yu AND SHANZHEN LU}

Abstract. In this paper, we study the generalized commutators of Sjölin type operator $T_{\alpha, A}^{a, m}$ defined by

$$
T_{\alpha, A}^{a, m} f(x)=\int_{\mathbb{R}^{n}} K_{\alpha}^{a}(x-y) \frac{R_{m}(A ; x, y)}{|x-y|^{m-1}} f(y) d y=\int_{\mathbb{R}^{n}} \frac{e^{i|x-y|^{a}}}{|x-y|^{\alpha}} \frac{R_{m}(A ; x, y)}{|x-y|^{m-1}} f(y) d y,
$$

where $R_{m}(A ; x, y)=A(x)-\sum_{|\alpha|<m} \frac{1}{\alpha !} D^{\alpha} A(y)(x-y)^{\alpha}$ with $m \in \mathbb{Z}^{+}$.

By using the scale changing method, we prove that if $D^{\gamma} A \in \dot{\Lambda}_{\beta}(0<\beta<1)$ with $|\gamma|=$ $m-1, m \geqslant 2$ or $A \in \dot{\Lambda}_{\beta} \quad(0<\beta<1)$ when $m=1, T_{\alpha, A}^{a, m}$ is bounded on $L^{p}\left(\mathbb{R}^{n}\right)$ for certain range of $p$.

Mathematics subject classification (2010): 47B38, 26D10, 47A30.

Keywords and phrases: Sjölin operator, generalized commutator, Lipschitsz space.

\section{REFERENCES}

[1] L. CARleson And P. SuÖLIn, Oscillatory integrals and a multiplier problem for the disc, Studia Math. 44, (1972), 287-299.

[2] J. Cohen And J. Gosselin, A BMO estimate for multilinear singular integrals, Illinois J. Math. 30, (1986), 445-464.

[3] R. A. Devore And R. C. Sharpley, Maximal functions measuring smoothness, Mem. Amer. Math. Soc. 47, (1984).

[4] S. JANSON, Mean oscillation and commutators of singular integral operators, Ark. Mat. 16, (1978), 263-270.

[5] J. F. LI, The boundedness of some operators and their commutator, PhD Thesis. Beijing: Beijing Normal University, (2005).

[6] J. F. LI, The estimates for sharp maximal functions of Multilinear Stongly Singular Integrals, Acta Math. Sin. (Engl. Ser.) 21, (2005), 1495-1508.

[7] J. F. Li AND S. Z. LU, Applications of the Scale Changing Method to Boundedness of Certain Commutators, Int. J. Appl. Math. Sci. 1, (2004), 1-12.

[8] J. F. Li AND S. Z. LU, $L^{p}$ estimates of multilinear operators on strongly singular integral operators, Nagoya Math. J. 181, (2006), 41-62.

[9] S. Z. LU, H. X. Wu AND P. Zhang, Multilinear Singular Integrals with Rough Kernel, Acta Math. Sin. (Engl. Ser.) 19, (2003), 51-62.

[10] S. Z. LU AND D. Y. YAN, $L^{p}$-boundedness of multilinear oscillatory singular integrals with Calderön-Zygmund kernel, Sci. in China, Ser. A, 45, (2002), 196-213.

[11] M. PALUSZÝNSKI, Characterization of the Besov spaces via the commutator operator of Coifman, Indiana Univ. Math. J., 44, (1995), 1-17.

[12] P. SJÖLIn, Convolution with Oscillating Kernels, Indiana Univ. Math. J., 30, (1981), 47-55.

[13] P. SJÖLIn, Convolution with Oscillating Kernels on $H^{p}$ spaces, J. Lond. Math. Soc. (2), 23, (1981), $442-454$. 
[14] E. M. Stein, Harmonic Analysis: Real-Variable methods, Orthogonality and Oscillatory Integrals, Princeton Univ. Press. N. J. 1993.

[15] X. X. TAO AND Y. P. WU, Boundedness for the multi-commutators of Calderón-Zygmund operators, J. Math. Inequal. 6, (2012), 655-672.

[16] C. WANG AND Z. F. Zhang, A new proof of Wu's theorem on vortex sheets, Sci. China Math. 55, (2012), 1449-1462.

[17] S. J. Wu, Mathematical analysis of vortex sheets, Comm. Pure Appl. Math. 59, (2006), 1065-1206.

[18] D. Y. YAN, Some problems on multilinear singular integral operators and multilinear oscillatory singular integral operators, PhD Thesis. Beijing: Beijing Normal University, 2001.

[19] X. YU AND X. X. TAO, Boundedness for a class of generalized commutators on $\lambda$-central Morrey space, Acta Math. Sin. (Engl. Ser.) 29, (2013), 1917-1926. 